Pacific Journal of Mathematics 


\title{
PULLING BACK BUNDLES
}

\author{
George R. KemPF
}

Let $D$ be an ample divisor on a smooth projective algebraic variety $X$. We will define the notion of a vector bundle $\mathscr{W}$ on $X$ to be strongly stable with respect to $D$. If $X$ has characteristic zero this definition is the same as the usual definition of stability. In general it implies stability.

Let $f: Y \rightarrow X$ be a finite morphism. Then we have the bundle $f^{*} \mathscr{W}$ on $Y$ which has the ample divisor $f^{-1} D$. If $\mathscr{W}$ is stable with respect to $D$, we will prove

THEOREM 1 (Characteristic zero). $f^{*} \mathscr{W}$ is the direct sum of stable bundles of the same slope with respect to $f^{-1} D$, i.e. $f^{*} \mathscr{W}$ is poly-stable.

Consider the special case of a finite morphism $f: \mathbb{P}^{n} \rightarrow \mathbb{P}^{n}$. For instance $f$ is given by raising the homogeneous coordinates to the $k$ th power. Then we have an essentially unique choice of $D$ and $f^{-1} D$. Our result is a strong version of the above problem. When rank $\mathscr{W}=2$ this is due to Barth [5].

THEOREM 2. If $\mathscr{W}$ is a strongly stable bundle on $\mathbb{P}^{n}$, then $f^{*} \mathscr{W}$ is strongly stable.

By Theorem 1 in characteristic zero we need only see that $f^{*} \mathscr{W}$ is indecomposable. One may apply this in particular to the MumfordHorrocks' bundle on $\mathbb{P}^{4}$ and thereby produce many other rank two bundles on $\mathbb{P}^{4}$ with larger Chern classes. See [6].

1. Stability and strong stability. Let $D$ be an ample divisor on a smooth projective variety $X$. Let $\mathscr{W}$ be a torsion-free coherent sheaf on $X$. The slope $\mu(\mathscr{W})=\operatorname{deg} \mathscr{W} / \operatorname{rank} \mathscr{W}$ where $\operatorname{deg} \mathscr{W}=$ $\left[c_{1}(\mathscr{W}) \cdot D^{\operatorname{dim} X-1}\right]$.

Then $\mathscr{W}$ is stable with respect to $D$ if $\mu(\mathscr{F})<\mu(\mathscr{W})$ for all nonzero coherent subsheaves $\mathscr{F} \varsubsetneqq \mathscr{W}$.

For strong stability we will assume that $\mathscr{W}$ is locally free. When $\mathscr{W}$ is strongly free if for all $0<i<\operatorname{rank} \mathscr{W}, \Gamma\left(X, \mathscr{L}^{\otimes-1} \otimes \bigwedge^{i} \mathscr{W}\right)=0$ for all invertible sheaves $\mathscr{L}$ on $X$ such that $\operatorname{deg} \mathscr{L} \geq i \mu(\mathscr{W})$. 
LEMMA 3. Strongly stable implies stable.

Proof. Let $0 \neq \mathscr{F} \varsubsetneqq \mathscr{W}$ be a coherent subsheaf of $\mathscr{W}$ of rank $i$. Then we have the obvious homomorphism $i: \bigwedge^{i} \mathscr{F} \rightarrow \bigwedge^{i} \mathscr{W}$. Let $\mathscr{L}$ be $\left(\bigwedge^{i} \mathscr{F} / \text { torsion }\right)^{\text {double dual }}$. Then $i$ induces an inclusion $\mathscr{L} \subset$ $\bigwedge^{i} \mathscr{W}$. Thus if $\mathscr{W}$ is strongly stable then $\operatorname{deg} \mathscr{L}<i \mu(\mathscr{W})$ but $\mu(\mathscr{F})=$ $\operatorname{deg} \mathscr{L} / i$. Thus $\mu(\mathscr{F})<\mu(\mathscr{W})$ and hence $\mathscr{W}$ is stable.

Thus one easily checks that stable means that no section of $\mathscr{L}^{\otimes-1} \otimes$ $\bigwedge^{i} \mathscr{W}$ satisfies the Plücker relations at the generic point $X$ if $\operatorname{deg} \mathscr{L} \geq$ $i \mu(\mathscr{W})$.

Next we will use some analysis.

Proposition 4. If $\operatorname{char}(X)=0$ then strongly stable $\Leftrightarrow$ stable.

Proof. Assume that $\mathscr{W}$ is stable. Let $\mathscr{W}$ be a Kähler metric with $c_{1}(D)$ as cohomology class. Then by the theorem of DonaldsonUhlenberg-Yau $\mathscr{W}$ admits a Kähler-Einstein metric. As mentioned in [4] $\bigwedge^{i} \mathscr{W}$ has a Kähler-Einstein metric of slope $i \mu(\mathscr{W})$. Thus by Kobayashi's theorem $\Lambda^{i} \mathscr{W}$ is the direct sum of stable bundles $\mathscr{F}_{*}$ of slope $i \mu(\mathscr{W})$. In particular each $\mathscr{F}_{*}$ does not contain an invertible sheaf $\mathscr{L}$ of $\operatorname{deg} \geq i \mu(\mathscr{W})$. Hence $\Lambda^{i} \mathscr{W}$ has the same property.

2. The proof of Theorem 1 . We will prove Theorem 1 by induction of dimension $X=n$. Let $h=\operatorname{dim} \mathscr{W}$.

If $n=1$ then $\mathscr{W}$ has a Hermitian-Einstein metric for some Hermitian metric $\omega_{X}$ on $X$. Thus $f^{*} \mathscr{W}$ has a Hermitian-Einstein metric for the degenerate metric $f^{*} w_{Z}$ on $Y$ which vanishes at the ramification points of $f$. Let $\mathscr{F} \subset f^{*} \mathscr{W}$ be a coherent sheaf of rank $f$, which we may assume is a subbundle as $Y$ is a smooth curve. Thus $\mathscr{L}=\Lambda^{h} \mathscr{F} \subset \Lambda^{h} f^{*} \mathscr{W}$ is a subbundle. Hence the curvature of $\mathscr{L}$ is pointwise smaller than that of $\Lambda^{h} f^{*} \mathscr{W}$.

We immediately conclude that $f^{*} \mathscr{W}$ is semi-stable. If the $\operatorname{deg} \mathscr{L}=$ slope $\Lambda^{h} f^{*} \mathscr{W}$, then $\mathscr{L}$ has a Hermitian-Einstein metric with respect to $f^{*} \omega_{X}$. Then we have a section of $\mathscr{L}^{\otimes-1} \otimes \bigwedge^{h} f^{*} \mathscr{W}$ corresponding to the inclusion but this sheaf has zero curvature. As usual we see that $\mathscr{F}$ is a direct summand of $f^{*} \mathscr{W}$.

For the inductive step let $X^{\prime}$ be a general hyperplane section of $X$ of large degree. Then $\left.\mathscr{W}\right|_{X^{\prime}}$ is stable by the restriction theorem of Mehta-Ramanathan [1]. By Bertini $f^{-1}\left(X^{\prime}\right)=Y^{\prime}$ is smooth. Trivially $f^{\prime}: Y^{\prime} \rightarrow X^{\prime}$ is finite. Then $f^{\prime *}\left(\left.\mathscr{W}\right|_{X^{\prime}}\right)=\left.f^{\prime *} \mathscr{W}\right|_{Y^{\prime}}$ is poly-stable. Say 
$\left.f^{\prime * \mathscr{W}}\right|_{Y^{\prime}}=\bigoplus \mathscr{V}_{i}^{\oplus n_{t}}$ where the $\mathscr{V}_{i}$ are non-isomorphic bundles with the same slope. It follows that

$$
\operatorname{End}\left(\left.f^{\prime *} \mathscr{W}\right|_{Y^{\prime}}\right)=\bigoplus \operatorname{End}_{\mathbb{C}}\left(\mathbb{C}^{\oplus n_{\iota}}\right)
$$

and each direct summand is given by a idempotent.

By Serre's vanishing theorem $\operatorname{End}\left(f^{*} \mathscr{W}\right) \rightarrow \operatorname{End}\left(\left.f^{*} \mathscr{W}\right|_{Y^{\prime}}\right)$ is an isomorphism because $Y^{\prime}$ has large degree. Thus we have a decomposition $f^{*} \mathscr{W}=\bigoplus \mathscr{W}_{i}^{\oplus n_{i}}$ which extends to the one above and this decomposition is independent of the choice of $Y^{\prime}$. Thus each $\mathscr{W}_{i}$ is stable and they have the same slope by the trivial direction of the reasoning of the restriction theorem. Thus Theorem 1 is here.

3. Endomorphisms of $\mathbb{P}^{n}$. Let $f: \mathbb{P}^{n} \rightarrow \mathbb{P}^{n}$ be a non-constant morphism. Then $f^{*} \mathscr{O}_{\mathbb{P}^{n}}(1)=\mathscr{O}_{\mathbb{P}^{n}}(k)$ where $k$ is positive. Now $f\left(x_{0}, \ldots, x_{n}\right)=\left(F_{0}(x), \ldots, F_{n}(X)\right)$ where $F_{0}, \ldots, F_{n}$ are homogeneous polynomials of degree $k$ with no common zero.

Let $i: k\left[Y_{0}, \ldots, Y_{n}\right] \rightarrow k\left[X_{0}, \ldots, X_{n}\right]$ be the homomorphism sending $Y_{i}$ to $F_{i}$. Then by the argument in invariant theory [3] we may conclude that $i$ is injective and $k\left[X_{0}, \ldots, X_{n}\right]$ is a free $k\left[Y_{0}, \ldots, Y_{n}\right]$-module with a basis $r_{1}, \ldots, r_{d}$ of homogeneous elements. This implies

LEMMA 5. (a) $f$ is a flat finite morphism.

(b) for all $l, f_{*}\left(\mathscr{O}_{\mathbb{P}^{n}}(l)\right)=\bigoplus_{m \in S(l)} \mathscr{O}_{\mathbb{P}^{n}}(m)$ where the finite set $S(l)$ satisfies

(c) $S(0)$ has only one non-negative element which is zero and $S(l)$ has non-negative elements if $l<0$.

Proof. The point (c) follows from (b) by looking to the isomorphism of global sections

$$
\Gamma\left(\mathbb{P}^{n}, \mathscr{O}_{\mathbb{P}^{n}}(l)\right)=\bigoplus_{m \in S(l)} \Gamma\left(\mathbb{P}^{n}, \mathscr{O}_{\mathbb{P}^{n}}(m)\right) .
$$

To prove (a) first note that $f$ is affine as $f^{-1}\left(Y_{i} \neq 0\right)=\left(F_{i} \neq 0\right)$ is affine. Thus (a) follows from (b). For (b) we compute

$$
\begin{aligned}
\Gamma\left(F_{i}\right) & \neq 0, \mathscr{O}_{\mathbb{P}^{n}}(l)=\left[k\left[X_{0}, \ldots, X_{n}\right]_{\left.F_{0}\right)}\right]_{\text {degree } l} \\
& =\bigoplus\left[r_{i}, k\left[Y_{0}, \ldots, Y_{n}\right]_{\left(X_{i}\right)}\right]_{\text {degree } l} \\
& =\bigoplus\left[r_{i}, k\left[Y_{0}, \ldots, Y_{n}\right]_{\left(X_{i}\right)}\right]_{\text {some degree depending on } r_{i}} .
\end{aligned}
$$

As this isomorphism is global (b) follows. 
4. The proof of Theorem 2. Let $f: \mathbb{P}^{n} \rightarrow \mathbb{P}^{n}$ be a non-constant morphism. Let $\mathscr{W}$ be a strongly stable vector bundle on $\mathbb{P}^{n}$ of slope $\mu$ with respect to $D$ where $D$ is a hyperplane section.

Now we want to prove that $f^{*} \mathscr{W}$ is strongly stable of slope $k \cdot \mu$ with respect to $D$ where $k D \sim f^{*} \mathscr{W}$. Let $\mathscr{L}$ be an invertible sheaf on $\mathbb{P}^{n}$ such that $\Gamma\left(\mathbb{P}^{n}, \mathscr{L}^{\otimes-1} \otimes \bigwedge^{j} f^{*} \mathscr{W}\right) \neq 0$ for $0<i<\operatorname{rank} \mathscr{W}$. Then we need to show that $\operatorname{deg} \mathscr{L}<i k \mu$. Let $\mathscr{L}=\mathscr{O}_{\mathbb{P}^{n}}(l)$.

Write $l=k r-s$ where $0 \leq s<k$. Then

$$
\begin{aligned}
\Gamma\left(\mathbb{P}^{n}, \mathscr{L}^{\otimes-1} \otimes \bigwedge^{i} f^{*} \mathscr{W}\right) & =\Gamma\left(\mathbb{P}^{n}, \mathscr{O}_{\mathbb{P}^{n}}(-s)\right) \otimes f^{*}\left(\mathscr{O}_{\mathbb{P}^{r}}(-r) \otimes \bigwedge^{i} \mathscr{W}\right) . \\
& =\Gamma\left(\mathbb{P}^{n}, \mathscr{O}_{\mathbb{P}^{r}}(-s)\right) \oplus\left(\mathscr{O}_{\mathbb{P}^{n}}(-r) \otimes \bigwedge^{i} \mathscr{W}\right) \\
& =\bigoplus_{m \in S(-s)} \Gamma\left(\mathbb{P}^{n}, \mathscr{O}_{\mathbb{P}^{n}}(-r m) \otimes \bigwedge^{i} \mathscr{W}\right) .
\end{aligned}
$$

As $\mathscr{W}$ is strongly stable we get $+r m<i \mu$ for some $m \in S(-s)$ where $m<0$ unless $s=0$ then $m \leq 0$. Thus $\operatorname{deg} \mathscr{L}=l=k r+s=$ $k(r+s / k) \leq k(r m) \leq k(i \mu)=i(k \mu)$ which is what we wanted.

5. Splitting of bundles. Let $\mathscr{W}$ be a bundle on $\mathbb{P}^{n}$. Then $\mathscr{W}$ is split if and only if $\mathscr{W}=\bigoplus \mathscr{O}_{\mathbb{P}^{n}}\left(l_{i}\right)$ for some $l_{i}$. Let $f: \mathbb{P}^{n} \rightarrow \mathbb{P}^{n}$ be a finite morphism.

LEMMA 6. $f^{*} \mathscr{W}$ is split iff $\mathscr{W}$ is.

Proof. The "if" part is trivial.

To prove the other way note that $H^{i}\left(\mathbb{P}^{n}, \mathscr{W}(i)\right)$ is a direct summand of $H^{i}\left(\mathbb{P}^{n}, f^{*} \mathscr{W}(k i)\right)$ by $\S 2$. Thus Horrocks' criterion [2] for $f^{*} \mathscr{W}$ implies the same condition for $\mathscr{W}$. Hence $\mathscr{W}$ is split if $f^{*} \mathscr{W}$ is.

\section{REFERENCES}

[1] V. B. Mehta and A. Ramanathan, Semistable sheaves on projective varieties and their restrictions to curves, Math. Ann., 258 (1982), 213-224.

[2] G. Horrocks, Vector bundles on the punctured spectrum of a local ring, Proc. London Math. Soc. (3), 14 (1958), 689-713.

[3] G. Kempf, Computing Invariants, in Invariant Theory (S. S. Koh), Lecture Notes in Math., vol. 1278, Springer-Verlag, New York, 1980, pp. 8-94.

[4] M. Lübke, Stability of Einstein-Hermitian vector bundle, Manuscripta Math., 42 (1983), 245-257.

[5] W. Barth, Some properties of stable rank-2 vector bundles on $\mathbb{P}^{n}$, Math. Ann., 226 (1977), 125-150.

[6] W. Decker and F. O. Schreyer, Pullbacks of the Horrocks-Mumford bundle, J. Reine Angew Math., 382 (1978), 215-220.

Received November 20, 1989. Partially supported by an NSF grant. 


\title{
PACIFIC JOURNAL OF MATHEMATICS EDITORS
}

V. S. VARADARAJAN

(Managing Editor)

University of California

Los Angeles, CA 90024-1555-05

HeRbert Clemens

University of Utah

Salt Lake City, UT 84112

F. Michael Christ

University of California

Los Angeles, CA 90024-1555

THOMAS ENRIGHT

University of California, San Diego

La Jolla, CA 92093
Nicholas ERcolani

University of Arizona

Tucson, AZ 85721

R. FINN

Stanford University

Stanford, CA 94305

VAUGHAN F. R. JONES

University of California

Berkeley, CA 94720

Steven Kerckhoff

Stanford University

Stanford, CA 94305

\section{C. MOORE}

University of California

Berkeley, CA 94720

Martin ScharlemanN

University of California

Santa Barbara, CA 93106

HAROLD STARK

University of California, San Diego

La Jolla, CA 92093

\section{ASSOCIATE EDITORS}
R. Arens
E. F. BECKENBACH
B. H. NeumanN
F. WoLF
K. YoshidA
(1906-1982)
(1904-1989)

\section{SUPPORTING INSTITUTIONS}

UNIVERSITY OF ARIZONA

UNIVERSITY OF BRITISH COLUMBIA

CALIFORNIA INSTITUTE OF TECHNOLOGY

UNIVERSITY OF CALIFORNIA

MONTANA STATE UNIVERSITY

UNIVERSITY OF NEVADA, RENO

NEW MEXICO STATE UNIVERSITY

OREGON STATE UNIVERSITY

\author{
UNIVERSITY OF OREGON \\ UNIVERSITY OF SOUTHERN CALIFORNIA \\ STANFORD UNIVERSITY \\ UNIVERSITY OF HAWAII \\ UNIVERSITY OF TOKYO \\ UNIVERSITY OF UTAH \\ WASHINGTON STATE UNIVERSITY \\ UNIVERSITY OF WASHINGTON
}

The Supporting Institutions listed above contribute to the cost of publication of this Journal, but they are not owners or publishers and have no responsibility for its content or policies.

\begin{abstract}
Mathematical papers intended for publication in the Pacific Journal of Mathematics should be in typed form or offset-reproduced (not dittoed), double spaced with large margins. Please do not use built up fractions in the text of the manuscript. However, you may use them in the displayed equations. Underline Greek letters in red, German in green, and script in blue. The first paragraph must be capable of being used separately as a synopsis of the entire paper. In particular it should contain no bibliographic references. Please propose a heading for the odd numbered pages of less than 35 characters. Manuscripts, in triplicate, may be sent to any one of the editors. Please classify according to the 1991 Mathematics Subject Classification scheme which can be found in the December index volumes of Mathematical Reviews. Supply name and address of author to whom proofs should be sent. All other communications should be addressed to the managing editor, or Elaine Barth, University of California, Los Angeles, California 90024-1555-05.

There are page-charges associated with articles appearing in the Pacific Journal of Mathematics. These charges are expected to be paid by the author's University, Government Agency or Company. If the author or authors do not have access to such Institutional support these charges are waived. Single authors will receive 50 free reprints; joint authors will receive a total of 100 free reprints. Additional copies may be obtained at cost in multiples of 50 .
\end{abstract}

The Pacific Journal of Mathematics (ISSN 0030-8730) is published monthly except for July and August. Regular subscription rate: $\$ 190.00$ a year (10 issues). Special rate: $\$ 95.00$ a year to individual members of supporting institutions.

Subscriptions, orders for numbers issued in the last three calendar years, and changes of address should be sent to Pacific Journal of Mathematics, P.O. Box 969, Carmel Valley, CA 93924, U.S.A. Old back numbers obtainable from Kraus Periodicals Co., Route 100, Millwood, NY 10546.

The Pacific Journal of Mathematics at P.O. Box 969, Carmel Valley, CA 93924 (ISSN 0030-8730) is published monthly except for July and August. Second-class postage paid at Carmel Valley, California 93924, and additional mailing offices. Postmaster: send address changes to Pacific Journal of Mathematics, P.O. Box 969, Carmel Valley, CA 93924.

PUBLISHED BY PACIFIC JOURNAL OF MATHEMATICS, A NON-PROFIT CORPORATION Copyright (C) 1992 by Pacific Journal of Mathematics 


\section{Pacific Journal of Mathematics}

Vol. 152, No. $2 \quad$ February, 1992

Edoardo Ballico, On the restrictions of the tangent bundle of the

Grassmannians

Edward Burger, Homogeneous Diophantine approximation in

$S$-integers

Jan Dijkstra, Jan van Mill and Jerzy Mogilski, The space of

infinite-dimensional compacta and other topological copies of $\left(l_{f}^{2}\right)^{\omega} \ldots 255$

Mike Hoffman, Multiple harmonic series ..................... 275

Wu Hsiung Huang, Superharmonicity of curvatures for surfaces of constant

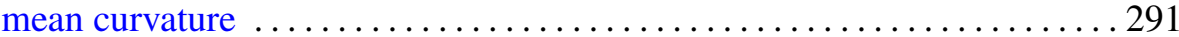

George Kempf, Pulling back bundles ......................... 319

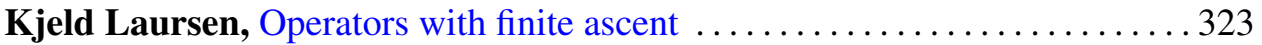

Andrew Solomon Lipson, Some more states models for link invariants . . . . 337

Xiang Yang Liu, Bloch functions of several complex variables .......... 347

Madabusi Santanam Raghunathan, A note on generators for arithmetic

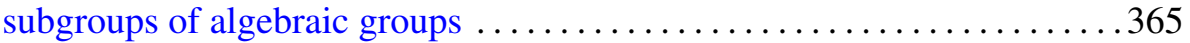

Marko Tadić, Notes on representations of non-Archimedean $\operatorname{SL}(n) \ldots \ldots 375$ 